\title{
TEM Sample Preparation Made Easier: Ar Ion Mill that Counts Reflected Light Fringes and Runs Recipes Automatically
}

\author{
Anahita Pakzad
}

Gatan Inc., R\&D Headquarters, Pleasanton, CA, USA

For samples to be suitable for observation in a transmission electron microscope (TEM), they need to be electron transparent (less than 100- $150 \mathrm{~nm}$ thick). Preparation of such specim ens is a tim e consuming procedure. There are multiple techniques such as electro-polishing, focused ion beam milling, wet polishing and Ar ion milling. Each of these methods is suitable for a sp ecific class of materials and has their own advantages and disadvantages. In each cas e, aside from selection of a suitable m ethod, one needs to develop a defined process, which is reproducible.

Broad beam Ar ion milling in Gatan's Pr ecision Ion Polishing System (PIPS) is one of the most common techniques that is used for preparation of electron transparent specimens of different classes of materials (e.g. sem iconductors, metals, ceramics, etc.). This m ethod consists of preparation of $3 \mathrm{~mm}$ disks (plan view or cross section stacks), mech anical polishing of these disks down to $\sim 100 \mu \quad \mathrm{m}$ thickness, dimple grinding the center of the disks to less than $15 \mu \mathrm{m}$, and lastly Ar ion $\mathrm{m}$ illing to electron transparency.

It is a common practice to watch the sam ple as it is being milled in the PIPS. First, because as it thins, milling parameters such as beam energy and angle need to be changed. Additionally it is very important to stop milling exactly at the right time to end up with a broad thin area, with the least amount of beam induced damage. In several cases, Newtonian light rings are used as a measure of the thickness of the sample. As the sam ple gets thinne $r$, these rings are formed by constructive/de structive interference between the light rays that reflect from top and bottom surfaces of the sample.

In the older generation of PIPS, the user controlled the $\mathrm{m}$ illing parameters manually using the front panel of the machine and watched the milling progress (growth of light fringes) through a $\mathrm{m}$ icroscope. Since Ar milling $\mathrm{m}$ ay require a few $\mathrm{m}$ inutes to many hours, the user had no option but $\mathrm{m}$ anual observation to determine the end point of the $p$ rocess. The new version of PIPS (PIPS II) is co mputer controlled. It inco rporates a touch screen gra phical user interface (GUI) and a digital zoom -in microscope. The touch screen controls all the parameters for operation of the tool, and is programmable to run recipes. The digital microscope is connected to a computer, which has Gatan's DigitalMicrograph software installed. This makes it possible to acquire and record images in addition to start/stop polishing or run recipes on the PIPS II directly from DigitalMicrograph.

DigitalMicrograph (DM) is an acquisition and im age processing software by Gatan, which is widely used in electron $m$ icroscopy community. This soft ware is based on a stra ightforward programming language, known as DM scripting. DM Scripting allows easy $m$ anipulation and evaluation of data, and automation of regularly perform ed tasks. Here we report on application of DM sc ripting function for automatic image acquisition, image processing and completion of PIPS II milling recipes to prepare a plan view and a cross section Si TEM sample.

In the cas e of the plan view sp ecimen, a two step recipe was progr ammed in the PIPS II, where the 
milling energy changed from $5 \mathrm{KeV}$ to $0.3 \mathrm{KeV}$ in the second step, keeping all the other param eters constant. Then a DM script was written in such way that DM runs the recipe. PIPS II records an optical image at each rotation during the milling process and as soon as DM detects light fringes in the image, the system pauses for the user to draw a line from the center of the fringes outwards. Milling continues with the first step parameters until the second blue fringe in the optical reflected light image is detected. DM command PIPS II to proceed to the second step in the recipe. Milling continues until the intensity of a specified number of pixels in th e central part of reflect ed light fringes reaches zero (perforation) and milling stops (Figure 1).

For the cross section sample, a 3 step recip e was programmed, where the milling energy ( $5 \mathrm{KeV}$ to 300 $\mathrm{eV}$ ) and milling angles $\left(7^{\circ}\right.$ to $\left.4^{\circ}\right)$ are reduced from one step to the next. The DM script for this sample was programmed to take an image from the sample before it starts milling and to ask the user to draw a line where the cross sec tion is in this im age. Next, the script starts running the first step of the recipe, and continues until the perforation is $10 \mu \mathrm{m}$ away from the glue line (c ross section). The second step in the recipe is started at this point and continues until the perforation is $2 \mu \mathrm{m}$ away from the glue line. Finally, the third s tep begins and mills the s ample at lo $\mathrm{w}$ angle an d low bea $\mathrm{m}$ energy until the perforation passes the glue line (Figure 2).
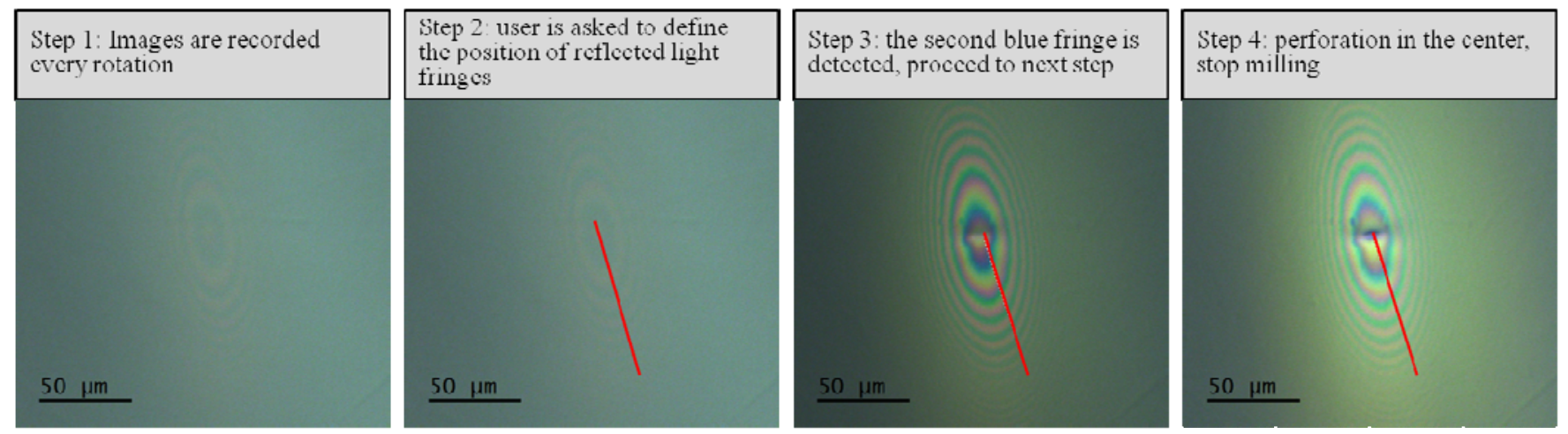

Figure 1. 4 steps programmed in the DM scrip t to automatically prepare a planar Si sample: 1) record an optical image each rotation, 2) fringes are detected, user is asked to define their location, 3) third blue fringe is detected, proceed to the next recipe step, 4) perforation is detected, stop milling.

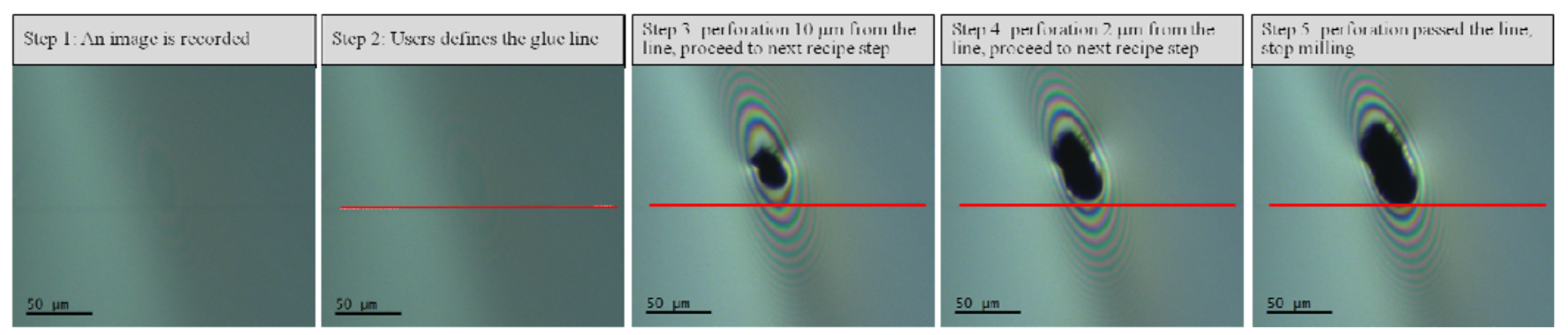

Figure 2. 5 steps programmed in the DM script to automatically prepare a cross section sample: 1) an optical image is recorded, 2) user is asked to define the glue line, 3) perforation in the sample is $10 \mu \mathrm{m}$ away from the line, proceed to the next step, 4) perforation in the sam ple is $2 \mu \mathrm{m}$ away from the line, proceed to the next step, 5) perforation passed the glue line, stop milling. 